\title{
Detection of a MicroRNA molecular signature of ultraviolet radiation in the superficial regions of melanocytic nevi on sun-exposed skin
}

\author{
Achim Bell ${ }^{1}$ - Diana Bell ${ }^{1}$ Nitin Chakravarti ${ }^{2} \cdot$ Junsheng Ma $^{3} \cdot$ Nicholas Henton $^{1} \cdot$ Victor G. Prieto $^{1}$
}

Received: 10 February 2018 / Revised: 13 May 2018 / Accepted: 14 May 2018 / Published online: 28 June 2018

(c) United States \& Canadian Academy of Pathology 2018

\begin{abstract}
How melanocytes transform into melanoma cells remains largely unknown. However, prolonged ultraviolet radiation exposure is linked with melanoma, and the DNA of melanomas arising in chronically sun-exposed skin is characterized by an elevated number of pyrimidine transitions, mainly $\mathrm{C}>\mathrm{T}$ (predominantly caused by ultraviolet $\mathrm{B}$ ), and transversions of GC $>$ TA or AT $>C G$ (caused by ultraviolet A over indirect mechanisms). Since ultraviolet penetrates mostly only the superficial dermis, we sought to determine the extent to which superficial and deep melanocytes of nevi in sun-exposed skin differ in their miRNA expression and consider the changes as likely secondary to ultraviolet radiation-induced damage. The differentially expressed miRNAs were analyzed for known potential oncomiRs or linked to potential oncogenes or tumor suppressors. Superficial and deep melanocytes were microdissected from the nevi of 14 patients. The suspensions were processed for hybridization to a ribonucleotide protection system with 2280 total probes, including 2256 miRNA probes targeting 2083 human miRNAs. A comprehensive analysis of all human miRNAs registered in miRBase 11.0 was performed using the HTG Molecular Diagnostic database. Statistical analysis of these data yielded for 14 samples a statistically relevant profile of 39 miRNA species at FDR $<0.1$ that were differentially expressed between superficial and deep melanocytes. Ingenuity Pathway Analysis based on the expression data of these 39 miRNAs suggested the gene transcripts AR, MDM2, SMAD2/3, and YBX1 as the most probable miRNA targets, which were validated at the protein level. Our findings suggest that superficial ultraviolet radiation-damaged melanocytes can be differentiated from deep melanocytes on the basis of the expression of 39 miRNAs, the most probable gene transcript and protein targets of which are AR, MDM2, SMAD2/3, and YBX1, with YBX1 expression validating the best the molecular signature in immunohistochemical analysis.
\end{abstract}

Electronic supplementary material The online version of this article (https://doi.org/10.1038/s41379-018-0088-5) contains supplementary material, which is available to authorized users.

Victor G. Prieto

vprieto@mdanderson.org

1 Department of Pathology and Dermatopathology, The University of Texas MD Anderson Cancer Center, 1515 Holcombe Blvd, Houston, TX 77030, USA

2 Center for Childhood Cancer \& Blood Diseases, Nationwide Children's Hospital, 700 Children's Drive, WA4112, Columbus, OH 43205, USA

3 Department of Biostatistics, The University of Texas MD Anderson Cancer Center, 1515 Holcombe Blvd., Houston, TX 77030, USA

\section{Introduction}

Melanocytes are derived from the neural crest during embryogenesis over two developmental pathways as melanoblasts and melanocyte precursor cells migrating to selected ectodermal sites, primarily to skin and central nervous system. There, melanocyte adult stem cells proliferate to differentiated melanocytes and migrate to separately reside within the epithelia, mostly the epidermis and hair follicles of the skin, but also in mucosal epithelium [1]. In contrast to this pathway, in melanocytic nevi, the differentiated melanocytes proliferate and mostly form nests or theques (small collections of melanocytes).

Melanocytic nevi represent a range of benign lesions expressing melanocytic phenotypes and have heterogeneous clinical and molecular characteristics [2]. Melanocytic nevi share many clinical features with melanoma and may be associated with increased risk of melanoma and other neoplasms and cancer syndromes. Melanocytic nevi 
develop as multifactorial processes under genetic and environmental influences. Depending on what patient's age the lesions occur, melanocytic nevi can be classified as either congenital or acquired. Benign melanocytic neoplasms are further classified as common acquired melanocytic nevi (not otherwise specified), dysplastic nevi (also called Clark nevi or nevi with architectural and cytologic atypia), Spitz nevi, BAP1-deficient nevi, congenital melanocytic nevi, and blue nevi, based on their dermoscopic, histological, and clinical characteristics [1, 3].

Both melanocytic nevi and melanoma share common driver mutations [1, 2]. Acquired melanocytic nevi mostly harbor oncogenic mutations in $B R A F$, which is the predominant oncogene associated with melanoma [2, 4-6]. Congenital melanocytic nevi harbor NRAS and BRAF mutations; blue nevi harbor GNAQ and GNA11 mutations; BAP1-deficient nevi carry mutations in BRAF and BAPl; and Spitz and atypical Spitz nevi often show point mutations in $H R A S$ and $B R A F$, as well as fusion genes of other kinases $[1-3,7,8]$. These primary driver mutations are thought to be essential for the development of nevi [2]. Nevus cell proliferation is stopped by different mechanisms of senescence programs, ultimately terminating nevus growth. The malignant progression of nevi can occur only upon the emergence of additional tumorigenic alterations, which may provide an escape from oncogeneinduced senescence [1, 2]. In addition, the majority of melanomas (70-80\%) appear to develop de novo from benign melanocytes without the intermediate step of nevus [9].

Solar ultraviolet radiation that reaches the earth's surface consists of 95-97\% ultraviolet A (wavelength 315-400 nm) and $3-5 \%$ ultraviolet $\mathrm{B}(280-315 \mathrm{~nm})$ and is an important natural mutagen, carcinogen, and immunosuppressor $[10,11]$. Of the ultraviolet radiation that reaches exposed human skin, ultraviolet B waves penetrate only the epidermis and the upper part of the dermis, to depths of $0.16-0.18 \mathrm{~mm}$, whereas only the longer ultraviolet A waves penetrate into the deeper dermis, to a depth of around $1 \mathrm{~mm}$ [11]. Ultraviolet radiation may directly cause cell damage, including genomic lesions in nuclear and mitochondrial DNA, through type I photosensitized reactions, or indirectly, via generation of reactive oxygen species in type II photosensitized reactions $[11,12]$. The degree and type of ultraviolet radiation-induced DNA damage depends primarily on the wavelength, with ultraviolet B causing the most serious and frequent damage. However, indirect DNA damage conveyed by type II photosensitized oxidation pathways in photocarcinogenesis, which is mostly caused by ultraviolet $\mathrm{A}$ and to a lesser extent by ultraviolet $\mathrm{B}$, accounts for an estimated $92 \%$ of melanomas [11]. To neutralize the damaging effects of ultraviolet radiation exposure, the affected epidermal and dermal cells start their ultraviolet radiation stress responses of immediate, inducible, and transient processes [13, 14]. These ultraviolet radiation responses, developed to preserve the genomic integrity, are achieved by the activation of DNA repair pathways and checkpoints for cell-cycle arrest. The failure of these responses in human skin may lead to inflammation, immune suppression, photoaging, and, in the worst case, skin carcinogenesis. The mechanisms and pathways by which melanocytes transform into melanoma cells are still mostly unknown. However, melanoma has been linked to intense ultraviolet radiation exposure, since melanomas arising in sun-exposed skin (mostly lentigo maligna-type melanoma) have DNA characterized by an elevated number of dipyrimidine transitions, mainly $\mathrm{C}>\mathrm{T}$ (by ultraviolet $\mathrm{B}$ ), and transversions of GC>TA or AT $>C G$ (by ultraviolet A) causing diverse cell responses [11, 15]. The impact of ultraviolet radiation from sun exposure on superficial and deep nevi may also cause changes in expression of miRNAs involved in regulation of pathways and mechanisms which may play important roles in the development of melanocytic nevi and melanoma. Interestingly, in contrast to other types of melanoma (particularly superficial spreading type), it is very infrequent to see an association of lentigo maligna melanoma and preexisting nevi.

Mature microRNAs (miRNAs) are small ( 22 nt) endogenous RNAs that can regulate gene expression by either the posttranscriptional induction of targeted mRNA degradation or the inhibition of mRNA-protein translation [16]. MiRNAs can modulate up to $60 \%$ of protein-coding genes, and thus are involved in the control of differentiation, apoptosis, proliferation, and many other important biological pathways [17]. Deregulated miRNA expression can promote, cause, or prevent carcinogenesis, with individual miRNAs, designated as oncomiRs, targeting gene transcripts to either drive or suppress tumor growth [16, 18]. Such altered miRNA expression profiles involved in the formation and growth of neoplastic tissues can be used to not only characterize human cancers by serving as markers of disease stage, aggressiveness, and other features but also discover hitherto unknown cancer-related regulatory networks and pathways [16, 19].

As a representation of chronic ultraviolet-induced changes in benign nevi, this study selected cases from sunexposed areas and diverse degrees of solar elastosis. By microdissecting those melanocytes located close or away from the epidermis, we considered that the changes identified between the two areas were likely to be at least in a significant proportion secondary to ultraviolet damage. The objectives of the present study were to determine the extent to which superficial and deep melanocytes of nevi in sun-exposed skin differ in their miRNA expression secondary to ultraviolet radiation-induced damage and whether these differentially expressed miRNAs are known 
potential oncomiRs or linked to potential oncogenes or tumor suppressors.

\section{Methods}

\section{Patients}

This study was performed in accordance with the principles of the Declaration of Helsinki and approved by MD Anderson's Institutional Review Board.

During routine sign-out sessions from one of the authors (VGP), nevi from sun-exposed areas (head, neck and upper extremities) were selected for the presence of solar elastosis and for a change in morphology from the lesion's superficial areas (close to the epidermis, with scattered large, pleomorphic melanocytes showing hyperchromatic nuclei) to its deeper areas (with smaller melanocytes within a delicate stroma). Overall, and supporting the diagnosis of nevus, the lesions appeared to mature with depth (diminution in melanin content and cellular size), did not show an expansile pattern of growth and in neither area were mitotic figures identified. We identified 14 patients (16 samples). The lesions were assessed to mark two different areas for microdissection for the superficial and deep regions. Due to the presence of focal cytologic atypia, in seven of the cases immunohistochemistry (HMB45 and a combination of anti-MART1 and anti-Ki67) was employed in a manner previously discussed [20]. In summary, the lesions had a low proliferation rate in the dermis, uniform expression of MART1 and decreased expression of HMB45 with depth in the dermis ("maturation").

\section{Whole miRNA transcriptome expression analysis}

Approximately $10 \mu \mathrm{m}$ thick sections of 16 formalin-fixed paraffin-embedded (FFPE) tissue blocks from sun-exposed nevi were cut, and then mounted on $2-\mu \mathrm{m}$ polyethylene napthalate membrane slides for microdissection under a Leica LMD6500 laser capture microscope (LCM) to collect samples for superficial and deep nevi tissue. Each sample was immediately suspended in EdgeSeq lysis buffer (HTG Molecular Diagnostics, Tucson, AZ). The suspensions were processed by HTG Molecular Diagnostics in a quantitative nuclease protection assay for hybridization with 2280 total RNA probes, including 2256 miRNA sequences targeting 2083 human miRNAs, using the HTG EdgeSeq array system. The raw data analysis, quality control, counts per million standardizations, and data normalization were performed according to the manufacturer's protocol (Supplemental Table 1).

\section{Statistical analysis}

All data analyses were based on normalized data. Samples with no data were excluded. When there were two measures for the same sample, the mean of the two measures was used. Deep nevus cell samples (labeled with a "D") were considered to be similar to standard dermal nevus cells, and superficial nevus cell samples (labeled with an "S") were considered to be possibly genetically altered cells. Incomplete "D" and "S" sample pairs were left out, and the remaining sample pairs were analyzed for transcript expression with paired two-sample t-tests, resulting in 15 paired samples for 2106 miRNA transcripts.

The statistical comparison analysis of superficial nevi versus deep nevi was performed using $\mathrm{R}$ version 3.3.1 (R Foundation for Statistical Computing, Vienna, Austria). To identify potential outlier samples, box plots were used to analyze the expression distribution of each sample; hierarchical cluster analysis was used to detect potential sample subgroups with similar expression patterns; and k-means clustering was performed. The three methods identified one outlier sample, thus reducing the number of total sample pairs to 14 .

The data of these 14 sample pairs were used to calculate raw $p$-values with paired $t$-tests, and adjusted $p$-values were calculated with the Benjamin and Hochberg method to control the false discovery rate (FDR). In total 39 miRNA transcripts with FDR values smaller than 0.1 were considered significant, representing an expected maximum false positive rate of $10 \%(3.9)$.

\section{Analysis of miRNAs for correlations with diseases, pathways, targets}

Using the program Ingenuity Pathway Analysis (IPA; QIAGEN Silicon Valley, Redwood, CA), we applied the data of the 39 significantly differentially expressed miRNA transcripts as mature miRNAs to a core analysis for disease correlations, and to miRNA pathway and target analyses.

\section{Immunohistochemistry of potential miRNA target proteins}

The data of the 39 differentially expressed miRNAs between superficial and deep melanocytes, yielded from our miRNA studies, were used for miRNA target analysis via IPA. On the basis of these IPA results, four prominent potential miRNA protein targets were selected, whose expression in FFPE nevi tissues we assessed with immunohistochemistry using antibodies against androgen receptor (AR; Santa Cruz), mouse double minute 2 homolog (MDM2; Santa Cruz), SMAD family member 2/3 (SMAD2/ 


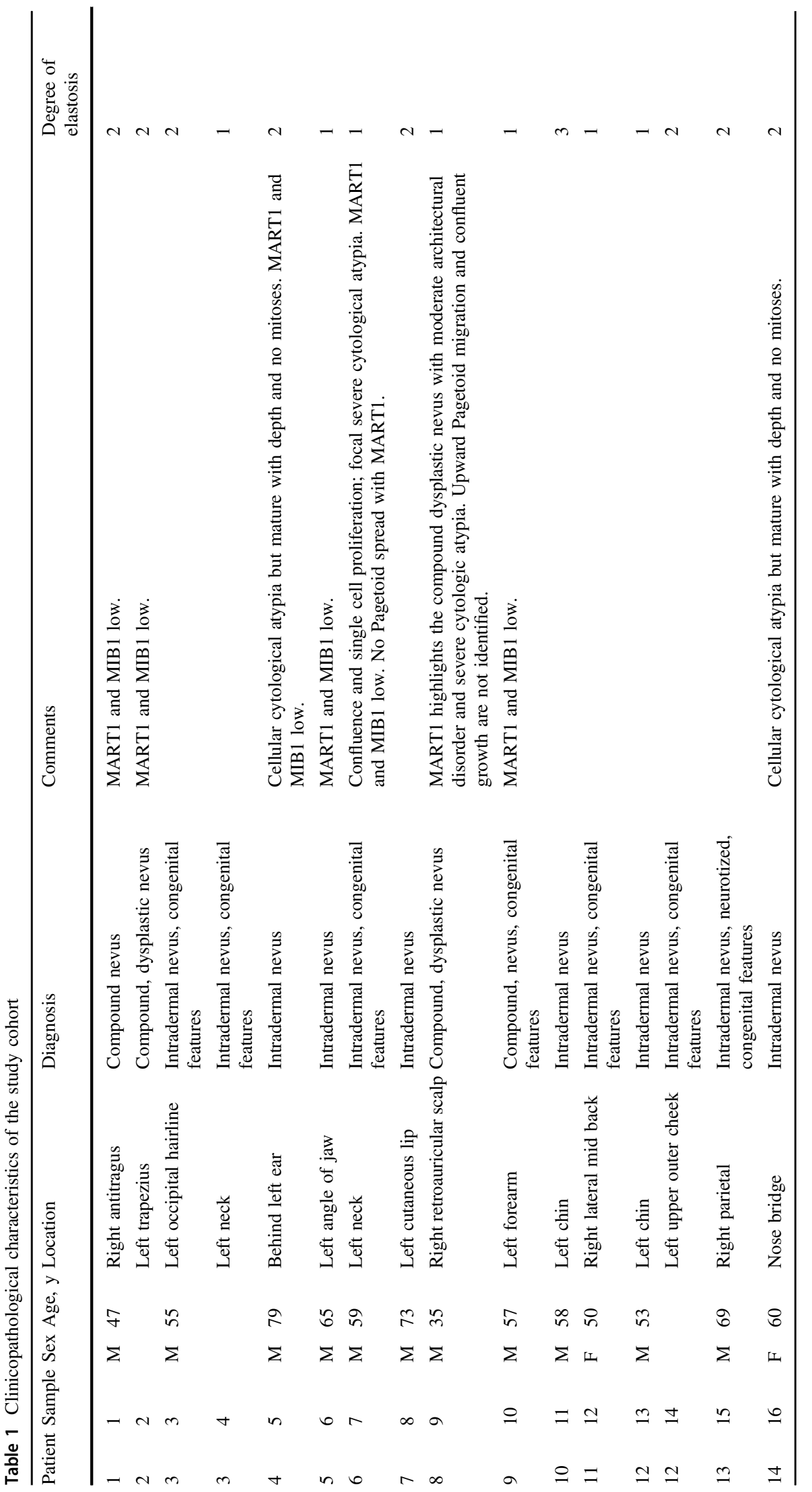



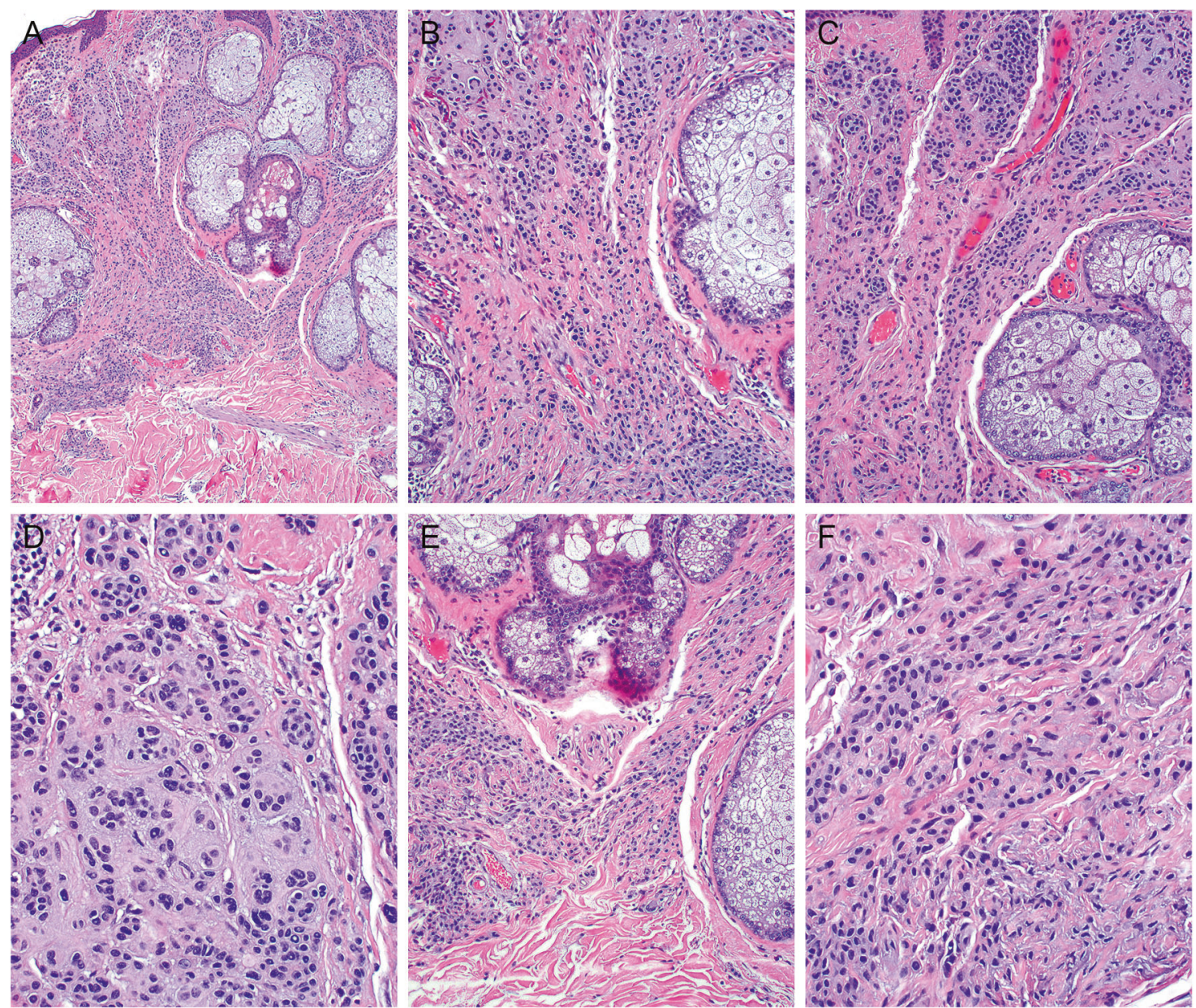

Fig. 1 Hematoxylin and eosin staining reveals the salient morphological features of analyzed nevi. a Compound nevus infiltrating between adnexal structures (magnification $\times 4$ ). b, c, e Superficial melanocytic elements transitioning into a deep melanocytic component

3; Santa Cruz), and Y box binding protein 1 (YBX1; Cell Signaling Technology).

\section{Results}

\section{Clinical and histological data}

Nevi diagnosis was established by an experienced dedicated dermatopathologist (VGP).

These lesions were selected during routine sign-out sessions and included nevi from sun-exposed areas in which there was an obvious change in morphology from the lesion's superficial areas (close to the epidermis, with large melanocytes showing hyperchromatic nuclei) to its deeper areas (with smaller melanocytes that appear to mature with depth). In neither area were mitotic figures identified. Solar elastosis was commonly identified in the papillary dermis. The clinical characteristics of the 14 patients are given in (magnification $\times 10$ ). d Enlarged superficial melanocytes with cytological atypia. f Deeper, smaller monotonous melanocytes (magnification $\times 20$ )

Table 1. Age at the time of diagnosis ranged from 35 to 79 years. Most patients (86\%) were men. The most common nevi location was the head and neck. The predominant histology was intradermal nevus $(n=11,6$ with congenital features, like perivascular and periadnexal pattern of growth; Fig. 1) followed by compound nevus ( $n=4,2$ with dysplastic features). Of note, patients 3 and 12 each provided 2 specimens of concurrent nevi from different locations. For nevi with more prominent cytologic atypia and dysplastic features, additional work-up with immunohistochemistry for MART1 and KI67 expression was employed to rule out the possibility of melanoma.

\section{Whole miRNA transcriptome expression analysis}

We identified a profile of 39 miRNAs that were differentially expressed between the superficial and deep melanocytes at FDRs of $<0.1$ (Table 2); none of these miRNA species belonged to any currently known oncomiRs. 
Table 2 Thirty-nine microRNAs (miRNAs) differentially expressed between sun-exposed superficial and deep nevi

\begin{tabular}{|c|c|c|c|c|}
\hline miRNA ID & IPA ID of miR family representative (same 7-nt seed sequence) & $\begin{array}{l}\text { Unadjusted } \\
p \text {-Value }\end{array}$ & FDR & LOG \\
\hline hsa-miR-582-5p & & $9.00 \mathrm{E}-05$ & 0.085 & 1.32 \\
\hline hsa-miR-181a-3p & hsa-miR-181a-1-3p & $1.03 \mathrm{E}-03$ & 0.087 & 0.47 \\
\hline hsa-miR-181d-5p & hsa-miR-181a-5p & $9.30 \mathrm{E}-04$ & 0.085 & 0.45 \\
\hline hsa-miR-106b-5p & hsa-miR-17-5p & $7.20 \mathrm{E}-04$ & 0.085 & 0.27 \\
\hline hsa-miR-1233-3p & & $9.30 \mathrm{E}-04$ & 0.085 & -0.66 \\
\hline hsa-miR-20b-3p & & $1.68 \mathrm{E}-03$ & 0.095 & -0.72 \\
\hline hsa-miR-6740-3p & & $1.44 \mathrm{E}-03$ & 0.092 & -0.86 \\
\hline hsa-miR-4465 & hsa-miR-26a-5p & $9.30 \mathrm{E}-04$ & 0.085 & -0.91 \\
\hline hsa-miR-6738-3p & & $1.41 \mathrm{E}-03$ & 0.092 & -0.93 \\
\hline hsa-miR-376b-5p & & $1.72 \mathrm{E}-03$ & 0.095 & -0.94 \\
\hline hsa-miR-3157-5p & & $1.30 \mathrm{E}-03$ & 0.092 & -1.02 \\
\hline hsa-miR-5195-3p & hsa-miR-145-5p & $5.10 \mathrm{E}-04$ & 0.085 & -1.03 \\
\hline hsa-miR-4730 & & $1.17 \mathrm{E}-03$ & 0.092 & -1.10 \\
\hline hsa-miR-7153-5p & hsa-miR-146a-5p & $8.50 \mathrm{E}-04$ & 0.085 & -1.11 \\
\hline hsa-miR-4724-3p & & $1.18 \mathrm{E}-03$ & 0.092 & -1.11 \\
\hline hsa-miR-6841-5p & & $1.71 \mathrm{E}-03$ & 0.095 & -1.11 \\
\hline hsa-miR-944 & & $1.49 \mathrm{E}-03$ & 0.093 & -1.12 \\
\hline hsa-miR-182-3p & & $3.60 \mathrm{E}-04$ & 0.085 & -1.12 \\
\hline hsa-miR-92a-2-5p & & $6.70 \mathrm{E}-04$ & 0.085 & -1.13 \\
\hline hsa-miR-489-5p & & $1.57 \mathrm{E}-03$ & 0.095 & -1.14 \\
\hline hsa-miR-1269a & & $9.80 \mathrm{E}-04$ & 0.086 & -1.14 \\
\hline hsa-miR-4691-5p & & $8.60 \mathrm{E}-04$ & 0.085 & -1.15 \\
\hline hsa-miR-593-3p & & $1.39 \mathrm{E}-03$ & 0.092 & -1.16 \\
\hline hsa-miR-6767-3p & & $1.84 \mathrm{E}-03$ & 0.099 & -1.19 \\
\hline hsa-miR-3616-3p & & $7.10 \mathrm{E}-04$ & 0.085 & -1.20 \\
\hline hsa-miR-6833-3p & & $7.80 \mathrm{E}-04$ & 0.085 & -1.27 \\
\hline hsa-miR-3677-5p & & $1.38 \mathrm{E}-03$ & 0.092 & -1.28 \\
\hline hsa-miR-548s & & $2.50 \mathrm{E}-04$ & 0.085 & -1.29 \\
\hline hsa-miR-7706 & & $1.40 \mathrm{E}-04$ & 0.085 & -1.29 \\
\hline hsa-miR-597-5p & hsa-miR-1970 & $1.43 \mathrm{E}-03$ & 0.092 & -1.29 \\
\hline hsa-miR-96-3p & & $4.50 \mathrm{E}-04$ & 0.085 & -1.31 \\
\hline hsa-miR-6828-5p & & $4.20 \mathrm{E}-04$ & 0.085 & -1.33 \\
\hline hsa-miR-372-5p & hsa-miR-295-5p & $3.80 \mathrm{E}-04$ & 0.085 & -1.34 \\
\hline hsa-miR-3193 & & $5.20 \mathrm{E}-04$ & 0.085 & -1.35 \\
\hline hsa-miR-6719-3p & & $5.60 \mathrm{E}-04$ & 0.085 & -1.37 \\
\hline hsa-miR-6814-3p & & $2.80 \mathrm{E}-04$ & 0.085 & -1.37 \\
\hline hsa-miR-5196-3p & & $1.20 \mathrm{E}-04$ & 0.085 & -1.38 \\
\hline hsa-miR-6742-3p & & $3.90 \mathrm{E}-04$ & 0.085 & -1.45 \\
\hline hsa-miR-4453 & & $6.70 \mathrm{E}-04$ & 0.085 & -1.47 \\
\hline
\end{tabular}

miR ID, 39 Homo sapiens micro RNA species were discovered after statistical analysis of the data received from quantitative nuclease protection assay with the HTG EdgeSeq array system. IPA ID of miR Family Representatives, refer to miR IDs, where IPA assigns different micro RNA species representing the same micro RNA family, defined by an identical $7 \mathrm{nt}$ seed/ consensus sequence

FDR false discovery rate values, samples with FDR values smaller 0.1 were considered as biological significant, LOG log 2 fold change of differential expression between deep nevi, which were considered similar to normal tissue versus superficial nevi, which were considered similar to atypical tissue 




Fig. 2 Hierarchical cluster analysis for similarity between superficial and deep nevi from 14 patients. A cluster dendrogram was generated from the hierarchical complete linkage clustering of 14 pairs of superficial nevi (S) and deep nevi (D) samples based on the expression data of 39 miRNA species from each nevus at FDRs of $<0.1$. The 2 main clusters comprised 1 group populated by 9 exclusively superficial samples (3.S, 2.S, 7.S, 13.S, 8.S, 14.S, 12.S, 5.S, and 11.S), which we assume represent atypical nevi, and the second group populated by the remaining 19 samples (14 deep and 5 superficial nevi), which we assume represent benign nevi

Hierarchical complete linkage clustering of the expression data of these 39 miRNA species for the 14 pairs of superficial melanocyte and deep melanocyte samples was performed to assess the sample groups for similarities and dissimilarities (Fig. 2). The main 2 clusters comprised 1 group populated by 9 exclusively superficial samples (3.S, 2.S, 7.S, 13.S, 8.S, 14.S, 12.S, 5.S and 11.S), which we assume represent cells more likely affected by ultraviolet radiation, and the second group populated by the remaining 19 samples (14 deep and 5 superficial nevi). As mentioned above it was interpreted that a significant component of the changes observed between the two groups were likely secondary to the effect of ultraviolet radiation (Fig. 2). Furthermore, when examining the results among the three types of nevi (predominantly intradermal, dysplastic, and with congenital pattern, there were no differences among the three types of nevi.

\section{Pathway analysis}

IPA suggested that the gene transcripts AR, MDM2, SMAD2/3, and YBX1 are the most probable miRNA targets (Fig. 3). The miRNAs hsa-miR-92a-2-5p, hsa-miR376b-5p, and hsa-miR-6738-3p possibly target AR. The miRNAs hsa-miR-5195-3p, hsa-miR-182-3p, hsa-miR548s, and hsa-miR-4453 possibly target MDM2, an inhibitor of tumor suppressor p53. The miRNA hsa-miR106b-5p possibly modulates the expression of SMAD2/3, which by itself might upregulate the expression of hsa-miR-181d-5p and downregulate the expression of hsamiR-372-5p. YBX1 is possibly involved in the downregulation of miR-96-3p.

\section{Biomarker analysis}

To validate the gene expression patterns detected by miRNA transcriptome expression analysis and IPA, we selected available antibodies to use immunohistochemistry to assess the protein expression of the possible targets.

YBX1 was expressed in 13 samples of superficial melanocytes; 5 of these samples had weak YBX1 expression. One sample was not available as the diagnostic tissue was exhausted. In contrast, YBX1 expression was absent in 10 samples and only weakly expressed in 3 samples of deep melanocytes (Fig. 4). The other three biomarkers (AR, MDM2, and SMAD2/3) did not show differences in expression between the two groups. A detailed analysis of the tissue samples and immunoreactivity with the biomarkers is shown in Table 3.

\section{Discussion}

Our results demonstrate that miRNA analysis of microdissected FFPE tissue provides insights into the molecular signature of ultraviolet radiation in the superficial regions of sun-exposed melanocytic nevi. Superficial melanocytes, which are supposedly affected by exposure to ultraviolet radiation, can be differentiated from deep melanocytes on the basis of the expression of 39 miRNAs, the most probable gene transcript and protein targets of which are AR, MDM2, SMAD2/3, and YBX1.

Our study has employed routine cases of nevi showing cytologic atypia in the superficial portions of lesions from sun-exposed areas. Therefore, some of the morphologic changes are likely to be secondary to ultraviolet light exposure. The cytologic atypia may resemble that seen in 


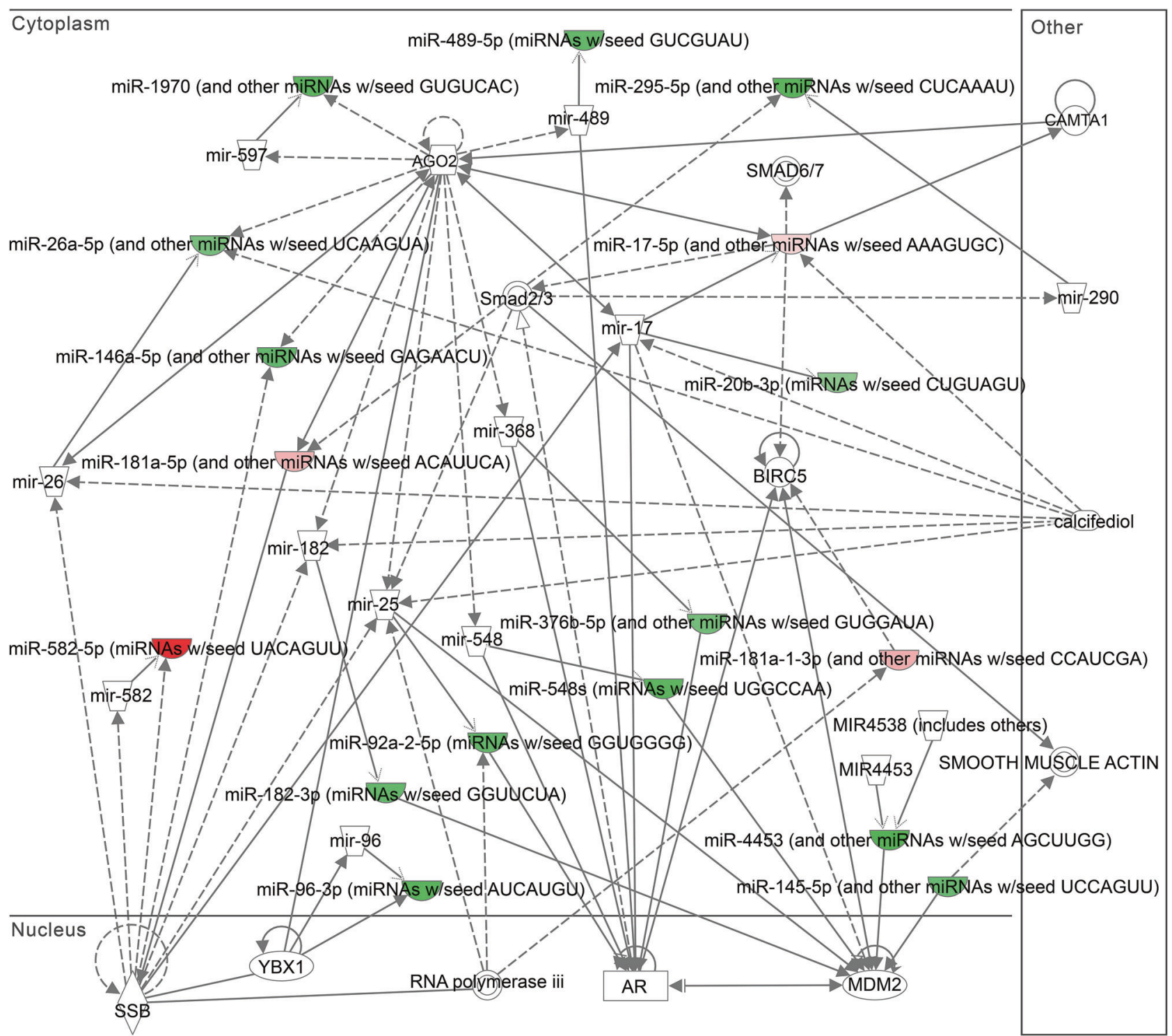

Fig. 3 Highest scoring pathway network created by the IPA program based on the data of 39 miRNAs differentially expressed between superficial and deep nevi (FDR<0.1). The IPA network focused on 17

"nevi with senescent atypia" [21] or nevi with "bizarre atypia" [22]. However, in both cases, there is no clear correlation of the presence of such atypical cells and depth within the lesion (both entities are described as having atypical cells dispersed through the entire depth of the nevi).

Of the 39 miRNAs in this profile, 35 (90\%) were downregulated in the superficial cells (Table 1). Interestingly, these miRNAs are generally associated with neoplastic tissues by promoting dedifferentiation to enable the epithelial-to-mesenchymal transition to cancer stem cells $[13,19]$. Although none of these miRNA species belonged to any well-known oncomiRs, some of the miRNAs share a 7-nt seed sequence with members of known oncomiR families. MiR-5195-3p shares the 7-nt seed sequence with
miRNAs associated with the network functions of "Organismal Injury and Abnormalities," "Reproductive System Disease," and "Cancer"

miR-145-5p, a member of the miR-145 family, which are known tumor suppressors in many cancers, including breast, colon, and bladder cancer [23-25]. MiR-7153-5p shares the 7-nt seed sequence with miR-146a-5p, which belongs to the miR-146 family, known to be involved in the inhibition of macrophage-induced apoptosis, angiogenesis activation [26], and metastasis formation in renal cell cancer [27]. And miR-106b-5p shares the 7-nt seed sequence with miR-17-5p, which belongs to the miR-17-92 cluster family, and is implicated in a wide variety of malignancies [28]; for example, miR-106b-5p activates cell proliferation and inhibits apoptosis in non-small cell lung cancer [29].

Relatively few studies have investigated ultraviolet radiation-induced miRNA signatures in melanocytic 


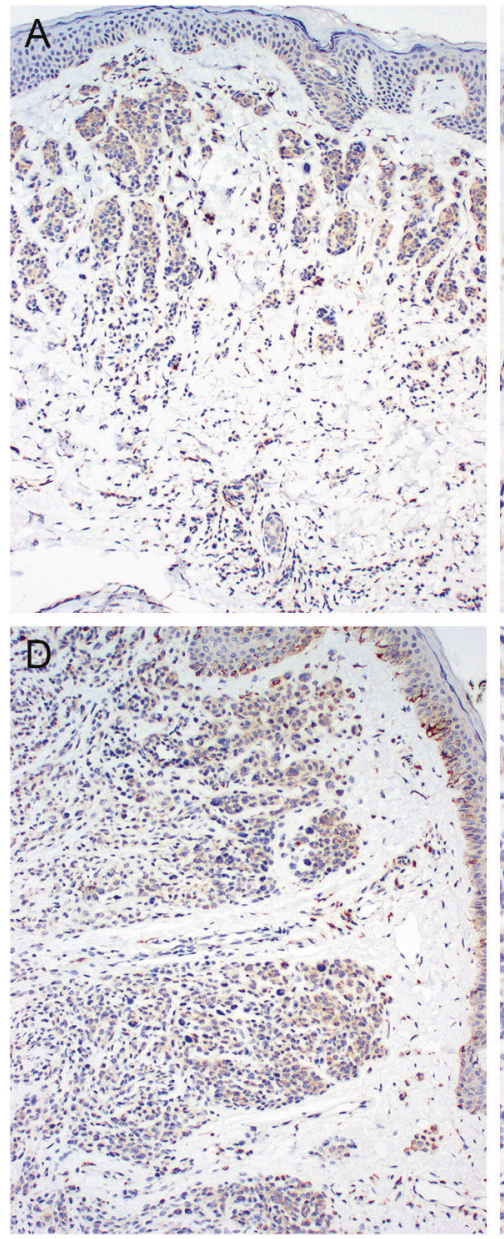

Fig. 4 Immunoperoxidase study with an anti-YBX1 antibody. Superficial melanocytes have a cytoplasmic and focal perinuclear distribution of YBX1 expression, which was not detected in deeper melanocytes. a, d Superficial melanocytes (magnification $\times 4$ ).

lesions. One of the first studies was similar to ours and investigated miRNAs that were differentially expressed between atypical melanocytic nevi (AN) and common acquired melanocytic nevi $(\mathrm{CN})$. The study identified a 36miRNA signature that prominently included the oncomiRs let- $7 \mathrm{a}$, let-7c, and miR-125b and revealed that miR-125b downregulation was correlated with a more malignant miRNA signature of AN compared with $\mathrm{CN}$ [16]. In contrast, we found very few similar miRNAs, i.e., the miR-92a family and three miRNA families identified by IPA as sharing the same 7-nt seed sequence: mir-145 (miR-51953p), miR-146a (miR-7153-5p), and miR-26a (miR-4465). The small overlap of our results with those of the previous study could be due to small but important differences in the selection of the experimental specimens for the AN and CN groups, which do not exactly match our selected groups of melanocytic nevi from superficial cells and deeper cells. It is unclear which areas of the nevi were harvested, i.e., superficial versus deep, and no correlation to the possible

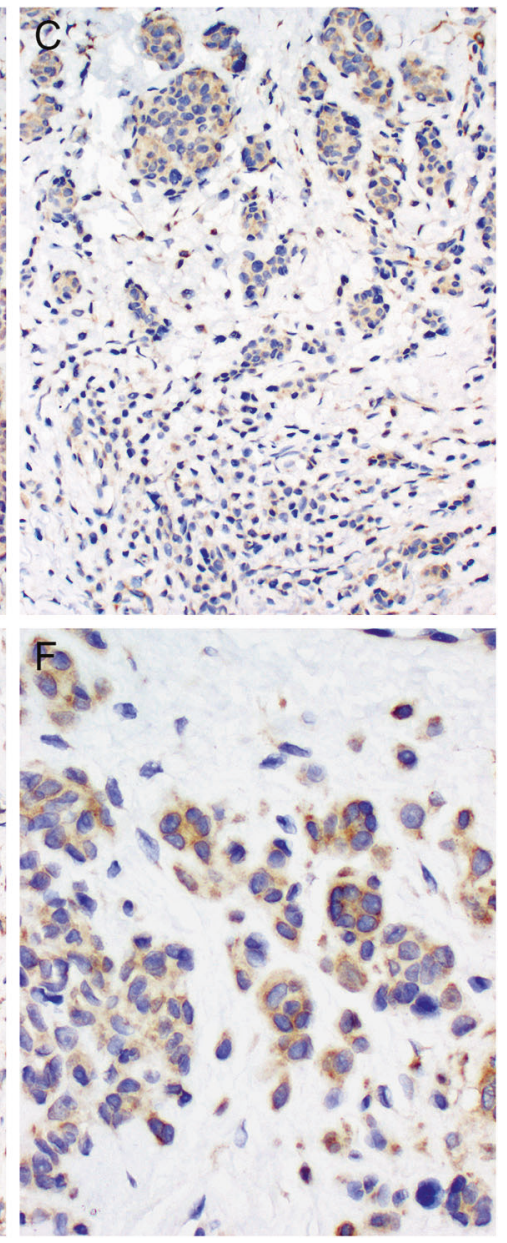

b, f Superficial melanocytes (magnification $\times 10$ ). c, e Transition between superficial and deep melanocytes (magnification $\times 10$ ). The samples are from patient $3 ; \mathbf{a}-\mathbf{c}$ shows sample 3 , and $\mathbf{b}-\mathbf{f}$ shows sample 4

amount of prior exposure to ultraviolet light. That the studies' findings had relatively few similarities could also be caused by differences in their methodologies; for example, the previous study used 4-6-mm punch biopsy specimens without further histopathology [16], whereas our specimens were selected on a LCM by an experienced pathologist for dissection and further processing.

In a previous study of intraepidermal melanocytes (not from preexistent nevi) obtained from women with a prior history of melanoma and comparing areas with or without irradiation with intermittent ultraviolet radiation, showed that most of the ultraviolet radiation-responsive miRNAs detected were downregulated, including miR-193b, miR342-3p, miR-186, miR-130a, and miR-146a. The only miRNA identified in that study that was included in our miRNA profile and found to be significantly affected by ultraviolet radiation was miR-106b-5p (represented in our IPA by the miRNA family miR-17-5p) [13]. In our study, this miRNA was one of only four upregulated miRNAS and 


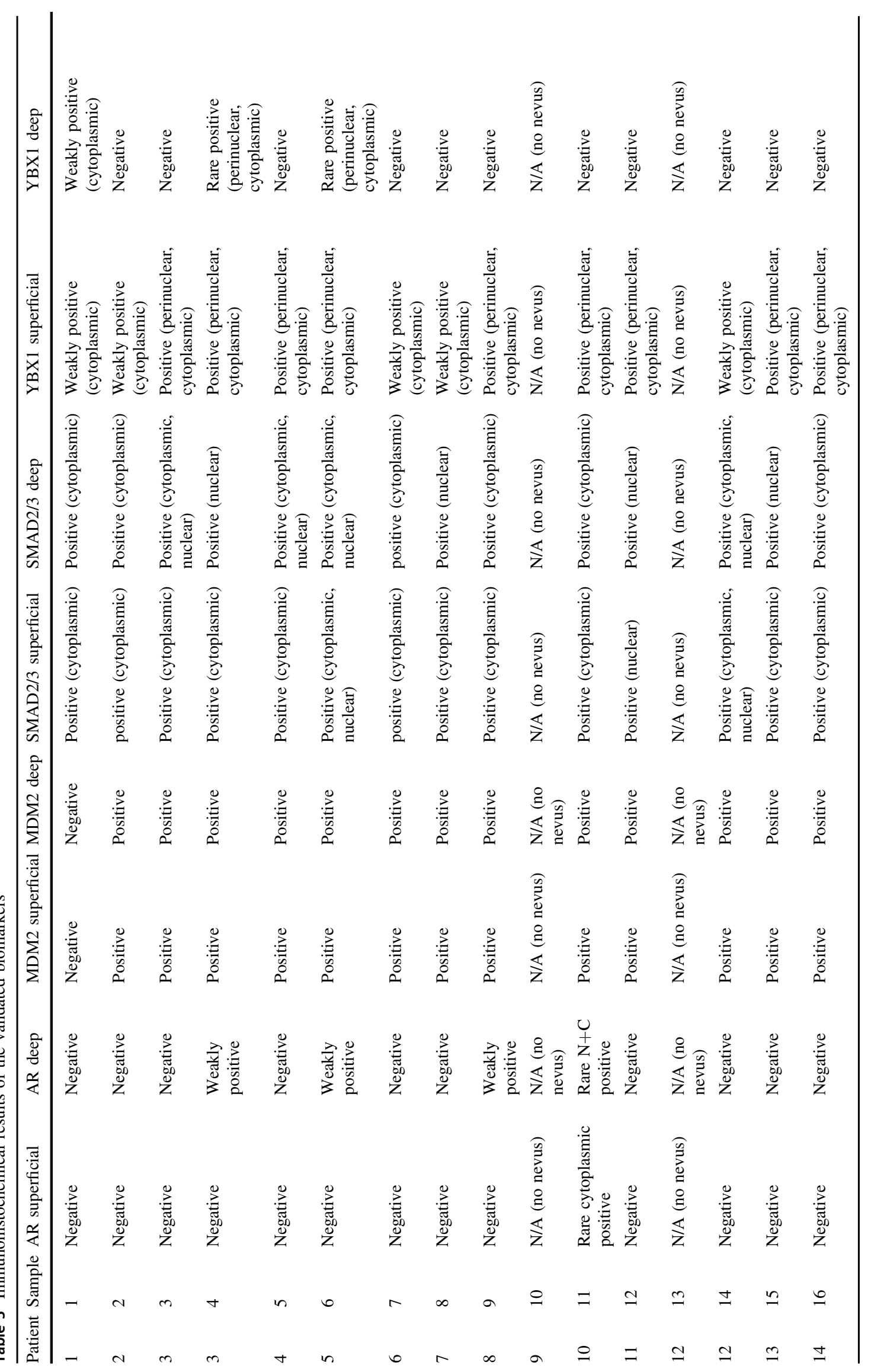


actually had the lowest upregulation value. In the previous study, miR-106b-5p had low upregulation in benign melanocytes but low downregulation in melanoma cells [13], which suggests that the superficial nevus cells exposed to ultraviolet radiation in the present study more closely resemble benign melanocytes than melanoma cells. The dissimilarity of the miRNA panels in our study and the previous study is probably due to the fact that the previous study investigated the effect of acute ultraviolet radiation on normal, intraepidermal melanocytes, whereas our study investigated the effects of chronic ultraviolet radiation exposure on the superficial regions of established, benign proliferations of melanocytes (i.e., nevi).

A previous review of miRNAs and photocarcinogenesis provides additional findings that give context to those of the present study. The miRNA signatures modulated in human skin cancers in that study include some of the miRNA families we identified [30]. For example, that study showed that, like the nevi in the present study, basal cell carcinoma has upregulated miR-181d and miR-17 (miR-106-5p) families and downregulated miR-145-5p (miR-5195-3p); however, miR-182-3p is upregulated in basal cell carcinoma but downregulated in nevi. In melanoma and in nevi, the miR-146a-5p (miR-7153-5p) family is downregulated. Interestingly, whereas the miR-145-5p (miR-5195-3p) family members are downregulated in basal cell carcinoma, squamous cell carcinoma, and nevi, they are upregulated in melanoma [30]. These findings indicate that the superficial nevi miRNA profile that we identified shares the same regulatory activity as 3 miRNA families in basal cell carcinoma, one miRNA family (i.e., miR-146a-5p, miR-7153$5 p$ ) in melanoma, and one miRNA family (miR-145-5p, miR-5195-3p) in both basal cell carcinoma and squamous cell carcinoma. This may indicate that superficial cell nevi share some small similarities with basal cell carcinoma but bear little similarity to squamous cell carcinoma and melanoma. The high dissimilarities of these miRNA profiles might correctly represent the differences between the superficial nevi cells and the neoplastic tissue cell samples discussed here and may even show that these groups have some small relatedness by sharing one or three of their signature miRNAs.

The results of the network analysis of our 39-miRNA panel indicate that the expressions of YBX1, AR, MDM2, and SMAD2/3 are correlated with the histological differences between superficial and deep nevi cells, with YBX1 expression showing the strongest correlation. YBX1, which encodes a multifunctional protein of the cold shock domain family that has broad nucleic acid binding properties, is involved in many cellular processes, including transcriptional and translational regulation, DNA repair, stabilization and processing of coding [31] and non-coding RNA, including miRNAs [32]. Extracellularly secreted YBX1 functions as a mitogen, cytosolic YBX1 acts in mRNA stabilization and translational regulation, and nuclear YBX1 functions in DNA repair and transcriptional regulation. In addition, the YBX1-mediated repression of miRNA has been observed in the nucleus, where it inhibits Drosha cleavage of pri-miRNA, as well as in the cytosol, where it inhibits Dicer cleavage of pre-miRNA [32]. Furthermore, aberrant YBX1 expression (primarily YBX1 upregulation) is associated with the tumorigenesis, growth, and chemoresistance of numerous different neoplastic tissues [31], and upregulated YBX1 expression increases the viability, migration, and invasion of melanoma cells [33]. On the basis of the results of these studies and the present study, we cautiously posit that the upregulation of the multifunctional YBX1 protein and its cytosolic and nuclear localization in superficial nevi may be related to activated DNA repair after ultraviolet radiation damage and to the YBX1mediated deregulation of miRNA processing increasing these cells' ability to transform into neoplasms. The deregulation of miRNA processing would downregulate miR-96-3p in particular but may also influence the formation of RNA/argonaute 2 complexes, i.e., RNA-induced silencing complexes [34].

In conclusion, our data suggest that the profile of the 39 miRNAs presented here defines modulators of atypical benign superficial nevi with increased potential to become malignant melanoma cells. Our data also suggest that the YBX1 protein might be a suitable biomarker of such atypical superficial melanocytes.

Acknowledgements We thank at The University of Texas MD Anderson Cancer Center, the Dermatopathology section of the Pathology Department and Dr. Jing Ning of the Department of Biostatistics for their support, the Laboratory of the Translational Molecular Pathology for facilitating the immunohistochemical study, and Mr. Joseph Munch from the Department of Scientific Publications, for his helpful editing input.

\section{Compliance with ethical standards}

Conflict of interest The authors declare that they have no conflict of interest.

\section{References}

1. Bastian BC. The molecular pathology of melanoma: an integrated taxonomy of melanocytic neoplasia. Annu Rev Pathol. 2014; 9:239-71.

2. Roh MR, Eliades P, Gupta S, et al. Genetics of melanocytic nevi. Pigment Cell Melanoma Res. 2015;28:661-72.

3. Rogers T, Marino ML, Raciti P, et al. Biologically distinct subsets of nevi. G Ital Dermatol Venereol. 2016;151:365-84.

4. Babapoor S, Wu R, Kozubek J, et al. Identification of microRNAs associated with invasive and aggressive phenotype in cutaneous melanoma by next-generation sequencing. Lab Invest. 2017;97:636-48. 
5. Dadras SS. Molecular diagnostics in melanoma: current status and perspectives. Arch Pathol Lab Med. 2011;135:860-9.

6. Howell PM Jr, Li X, Riker AI, et al. MicroRNA in melanoma. Ochsner J. 2010;10:83-92.

7. Edwards PA. Fusion genes and chromosome translocations in the common epithelial cancers. J Pathol. 2010;220:244-54.

8. Mertens F, Johansson B, Fioretos T, et al. The emerging complexity of gene fusions in cancer. Nat Rev Cancer. 2015;15:371-81.

9. Cymerman RM, Shao Y, Wang K, et al. De Novo vs nevusassociated melanomas: differences in associations with prognostic indicators and survival. J Natl Cancer Inst. 2016;108:1-9. Published by Oxford University Press

10. Sun X, Kim A, Nakatani M, et al. Distinctive molecular responses to ultraviolet radiation between keratinocytes and melanocytes. Exp Dermatol. 2016;25:708-13.

11. Valejo Coelho MM, Matos TR, Apetato M. The dark side of the light: mechanisms of photocarcinogenesis. Clin Dermatol. 2016;34:563-70.

12. Baptista MS, Cadet J, Di Mascio P, et al. Type I and Type II photosensitized oxidation reactions: guidelines and mechanistic pathways. Photochem Photobiol. 2017;93:912-9.

13. Sha J, Gastman BR, Morris N, et al. The response of microRNAs to solar UVR in skin-resident melanocytes differs between melanoma patients and healthy persons. PLoS ONE. 2016;11: e0154915.

14. Syed DN, Khan MI, Shabbir M, et al. MicroRNAs in skin response to UV radiation. Curr Drug Targets. 2013; 14:1128-34.

15. Mitchell D, Fernandez A. The photobiology of melanocytes modulates the impact of UVA on sunlight-induced melanoma. Photochem Photobiol Sci. 2012;11:69-73.

16. Holst LM, Kaczkowski B, Glud M, et al. The microRNA molecular signature of atypic and common acquired melanocytic nevi: differential expression of miR-125b and let-7c. Exp Dermatol. 2011;20:278-80.

17. Catalanotto C, Cogoni C, Zardo G. MicroRNA in control of gene expression: an overview of nuclear functions. Int $\mathrm{J}$ Mol Sci. 2016;17:1-17

18. He L, Thomson JM, Hemann MT, et al. A microRNA polycistron as a potential human oncogene. Nature. 2005;435:828-33.

19. Lu J, Getz G, Miska EA, et al. MicroRNA expression profiles classify human cancers. Nature. 2005;435:834-8.

20. Prieto VG, Shea CR, Reed JA. Anathema or useful? Application of immunohistochemistry to the diagnosis of melanocytic lesions. Pathology of challenging melanocytic neoplasms diagnosis and management. New York: Springer; 2015. p. 35-41. Edited by Shea CR, Reed JA and Prieto VG.

21. Boyd AS, Chen SC, Shyr Y. Intradermal nevi with atypical nuclei in the elderly: the senescent nevus. J Am Acad Dermatol. 2015;73:500-6.

22. McGovern VJ. Melanoma: histological diagnosis and prognosis. New York: Raven Press; 1983.

23. Lei C, Du F, Sun L, et al. miR-143 and miR-145 inhibit gastric cancer cell migration and metastasis by suppressing MYO6. Cell Death Dis. 2017;8:e3101.

24. Wu H, Xiao Z, Wang K, et al. MiR-145 is downregulated in human ovarian cancer and modulates cell growth and invasion by targeting p70S6K1 and MUC1. Biochem Biophys Res Commun. 2013;441:693-700.

25. Jiang Z, Zhang Y, Cao R, et al. miR-5195-3p inhibits proliferation and invasion of human bladder cancer cells by directly targeting oncogene KLF5. Oncol Res. 2017;25:1081-7.

26. Simanovich E, Brod V, Rahat MM, et al. Function of miR-146a$5 p$ in tumor cells as a regulatory switch between cell death and angiogenesis: macrophage therapy revisited. Front Immunol. 2017;8:1931

27. Wotschofsky Z, Gummlich L, Liep J, et al. Integrated microRNA and mRNA signature associated with the transition from the locally confined to the metastasized clear cell renal cell carcinoma exemplified by miR-146-5p. PLoS ONE. 2016;11:e0148746.

28. Sengupta D, Govindaraj V, Kar S. Alteration in microRNA-17-92 dynamics accounts for differential nature of cellular proliferation. FEBS Lett. 2018;592:446-58.

29. Wei K, Pan C, Yao G, et al. MiR-106b-5p promotes proliferation and inhibits apoptosis by regulating BTG3 in non-small cell lung cancer. Cell Physiol Biochem. 2017;44:1545-58.

30. Syed DN, Lall RK, Mukhtar H. MicroRNAs and photocarcinogenesis. Photochem Photobiol. 2015;91:173-87.

31. Matsumoto K, Bay BH. Significance of the Y-box proteins in human cancers. J Mol Genet Med. 2005;1:11-7.

32. Wu SL, Fu X, Huang J, et al. Genome-wide analysis of YB-1RNA interactions reveals a novel role of YB-1 in miRNA processing in glioblastoma multiforme. Nucleic Acids Res. 2015;43:8516-28.

33. Jia J, Zheng Y, Wang W, et al. Antimicrobial peptide LL-37 promotes YB-1 expression, and the viability, migration and invasion of malignant melanoma cells. Mol Med Rep. 2017;15:240-8.

34. Hock J, Weinmann L, Ender C, et al. Proteomic and functional analysis of Argonaute-containing mRNA-protein complexes in human cells. EMBO Rep. 2007;8:1052-60. 